\title{
The relationship between severe maternal morbidity and psychological health symptoms at 6-8 weeks postpartum: a prospective cohort study in one English maternity unit
}

Marie Furuta ${ }^{1 *}$, Jane Sandall ${ }^{2}$, Derek Cooper ${ }^{2}$ and Debra Bick ${ }^{3}$

\begin{abstract}
Background: The incidence of severe maternal morbidity is increasing in high-income countries. However, little has been known about the impact on postnatal morbidity, particularly on psychological health outcomes. The objective of this study was to assess the relationship between severe maternal morbidity (ie. major obstetric haemorrhage, severe hypertensive disorders or intensive care unit/obstetric high dependency unit admission) and postnatal psychological health symptoms, focusing on post-traumatic stress disorder (PTSD) symptoms at 6-8 weeks postpartum.
\end{abstract}

Method: A prospective cohort study was undertaken of women who gave birth over six months in 2010 in an inner city maternity unit in England. Primary outcomes were prevalence of PTSD symptoms namely: 1) intrusion and 2) avoidance as measured using the Impact of Event Scale at $6-8$ weeks postpartum via a self-administered postal questionnaire. Secondary outcomes included probable depression. Data on incidence of severe maternal morbidity were extracted from maternity records. Multivariable logistic regression analysis examined the relationship between severe maternal morbidity and PTSD symptoms taking into account factors that might influence the relationship.

Results: Of women eligible to participate ( $n=3509), 52 \%$ responded. Prevalence of a clinically significant level of intrusion and avoidance were 6.4\% $(n=114)$ and $8.4 \%(n=150)$ respectively. There was a higher risk of PTSD symptoms among women who experienced severe maternal morbidity compared with women who did not (adjusted OR $=2.11$, $95 \% \mathrm{Cl}=1.17-3.78$ for intrusion; adjusted $\mathrm{OR}=3.28,95 \% \mathrm{Cl}=2.01-5.36$ for avoidance). Higher ratings of reported sense of control during labour/birth partially mediated the risk of PTSD symptoms. There were no statistically significant differences in the prevalence or severity of symptoms of depression.

Conclusion: This is one of the largest studies to date of PTSD symptoms among women who had recently given birth. Findings showed that an experience of severe maternal morbidity was independently associated with symptoms of PTSD. Individually tailored care that increases women's sense of control during labour may be a protective factor with further work required to promote effective interventions to prevent these symptoms. Findings have important implications for women's health and the content and organisation of maternity services during and after the birth.

Keywords: Stress disorders, Post-traumatic, Pregnancy complications, Puerperal disorders, Postnatal care, Cohort studies

\footnotetext{
* Correspondence: furuta.marie.8r@kyoto-u.ac.jp

${ }^{1}$ Department of Human Health Sciences, Graduate School of Medicine, Kyoto University, 53 Shogoin Kawara-cho, Sakyo-ku, Kyoto City, Kyoto 606-8507, Japan

Full list of author information is available at the end of the article
} 


\section{Background}

As maternal mortality has declined in the UK, severe maternal morbidity is increasingly referred to as a more useful indicator of the safety and quality of maternity care [1]. However, little is known about the impact of severe maternal morbidity on women's postnatal health. Studies have shown that the level of intervention during labour and birth affects the risk of experiencing fear and anxiety [2]. The combination of experiencing a life-threatening complication and necessary medical interventions may culminate in maternal psychological and physical morbidity [3]. This may in turn 'trigger' post-traumatic stress disorder (PTSD) in the postnatal period [4-6].

PTSD is a condition that can develop after a person has experienced or witnessed a highly traumatic event. PTSD involves three symptom clusters: intrusive recollection, avoidance and hyperarousal [7]. The Diagnostic and Statistical Manual of Mental Disorders (DSM-IV) recognises that an individual's perception of threat and their response to an event critically affects subsequent development of PTSD [7]. Earlier studies have shown that PTSD affects approximately $2 \%$ to $6 \%$ of women at around six weeks following even spontaneous childbirth (i.e., full-term pregnancy with healthy outcome) using a range of measures [8,9]. A recent systematic review [10] identified the possibility that women's experiences of maternal morbidity could trigger PTSD symptoms indirectly through a third factor such as infant condition (e.g. prematurity, death) and a woman's perceived loss of control during labour and birth. However, due to methodological limitations including small sample sizes and unclear definition of severe maternal morbidity, the relationship between severe maternal morbidity and PTSD and possible mechanisms underlying the relationship could not be fully explained, leading to an evidence gap to support timely and appropriate care.

PTSD during the postpartum period is an important public health issue because of the longer-term negative impact of maternal mental health problems on child development [11-13] including impaired mother-infant relationship [14,15], delayed intellectual development $[16,17]$ and psychiatric disorder in children [18]. Studies showed intrusion symptoms (eg. flashback memory or re-experiencing a traumatic birth) may affect women's ability to adapt to motherhood and their relationships with others [2]. The experience of avoidance symptoms during the postnatal period may also impair a woman's ability to talk about and process the trauma, leading to social isolation [2], with potential implications for her decisions about infant feeding $[19,20]$. Long-term maternal morbidity, if not identified or appropriately managed at an early stage, could also increase use of health care services by women and their families $[21,22]$. We therefore assessed the impact of severe maternal morbidity on postnatal maternal health among women who had recently given birth, focusing particularly on post-traumatic stress disorder (PTSD) at 6-8 weeks when routine UK maternity care provision ends. Objectives included to: 1) identify the prevalence of postnatal PTSD symptoms and other psychological outcomes among women who gave birth, 2) compare the rates of PTSD symptoms and other psychological outcomes in women who had severe maternal morbidity and those who did not; and 3) examine the relationship between severe maternal morbidity and PTSD symptoms taking into account factors that might influence the relationship.

\section{Methods}

We undertook a prospective cohort study with severe maternal morbidity as the exposure and PTSD symptoms and postnatal depression symptoms as outcomes. After reviewing the definitions of severe maternal morbidity used in population-based studies in the UK and other high income countries [1,23-26], we selected two approaches to define this: disease-based and managementbased. Disease-based definitions included major obstetric haemorrhage and severe pre-eclampsia/eclampsia/HELLP syndrome (a syndrome involving haemolysis, elevated liver enzymes low platelets). Management-based definitions included admission to intensive care unit (ICU) or obstetric high dependency unit (HDU). Since almost all maternal complications after giving birth would be managed in the HDU in the study site, there was a considerable overlap between the two groups. However, including HDU admission in the management based group allowed us to identify less frequent types of severe maternal morbidity that would not be included in the disease based group. Women who had at least one episode of severe maternal morbidity (i.e. major obstetric haemorrhage, severe pre-eclampsia/eclampsia, HELLP syndrome, or ICU/HDU admission) were defined as having experienced severe maternal morbidity, while the remaining women were considered not to have experienced this.

\section{Primary outcomes}

Our primary outcome was the prevalence of PTSD symptoms measured by 1 ) intrusion and 2) avoidance at 6-8 weeks using the Impact of Event Scale (IES) [27]. The IES is validated in general populations and is one of the most widely used self-report scales to measure PTSD symptoms in postnatal populations $[28,29]$. The IES only measures two of the three PTSD symptoms (intrusion and avoidance but not hyperarousal). Earlier studies of PTSD symptoms in postnatal populations [30] suggested that predictors or contributing factors of intrusion and avoidance symptoms might not necessarily be the same. Therefore, in this study, intrusion and avoidance were examined separately as primary outcomes. Hyperarousal 
symptoms were not included because some symptoms (e.g., difficulty in falling or staying asleep, difficulty concentrating or irritability) are difficult to distinguish from a normal state in the postpartum period [31] and inclusion could be counterproductive.

The IES includes 15 items, measuring the frequency of symptoms of intrusion (seven items) and avoidance (eight items) during the past week. Women were asked to report how often during the previous week they had experienced symptoms of distress related to an event or experience during their labour, the birth of their baby, or immediately after the birth (within 24 hours) that made them feel anxious and frightened. The items were scored on a four point scale: not at all $(=0)$, rarely $(=1)$, sometimes $(=3)$ or often $(=5)$ [27]. Using a standard commonly used cut-off, scores of " 20 or more" in subscales (intrusion subscale score of 20 or more, or avoidance subscale score of 20 or more) were defined as a clinically significant level of distress [32], p.722. Based on the current study, the internal consistency of intrusion and avoidance with Cronbach's alpha was 0.87 and 0.86 , respectively.

\section{Secondary outcomes}

As individuals who suffer from PTSD may experience several symptoms at the same time or over a period of time, we included other indicators of PTSD symptoms as secondary outcomes-specifically, the prevalence of either intrusion or avoidance (a total score of 20 or more on either IES subscales) and the prevalence of both intrusion and avoidance (a total score of 20 or more on both subscales). Other secondary outcomes relevant to psychological health included continuous measures of symptoms of PTSD using IES scores and probable major depression based on a score of 13 or more on the Edinburgh Postnatal Depression Scale (EPDS) [33], with severity based on a high EPDS score. Studies in the UK have shown that using a threshold EPDS score of 12/13 in the sixth week postnatal, that sensitivity ranges from $68 \%$ to $95 \%$ and specificity from $78 \%$ to $96 \%$ compared to a diagnosis of major depression following psychiatric interview [33-35].

We also included routine and non-routine consultations with healthcare professionals, breastfeeding practice and general health as secondary outcomes, which will be reported elsewhere.

\section{Other variables}

Other variables that might influence the relationship between severe maternal morbidity and psychological outcomes were defined as potential confounders, mediators and effect modifiers.

\section{Potential confounders}

Confounders are variables which are associated with both the risk factor and causally related to the outcome
[36]. They may cause distortion in the effect of exposure of interest because "the effect of extraneous factors is mistaken for or mixed with the actual exposure effect (which may be null)" [37], p.120.

Following a systematic review [10], we included sociodemographic characteristics (maternal age, parity, ethnicity, educational qualification and an Index of Multiple Deprivation (IMD) [38]) and pre-existing health conditions (BMI as a measure of the level of overweight or obesity, and self-reported mental health history identified prior to giving birth) as potential confounders. Although a number of maternal health conditions prior to pregnancy may impact on a woman's experiences of severe maternal morbidity [39-42], BMI and self-reported mental health history were selected because they are not only associated with severe maternal morbidity, but may be potential risk factors for psychiatric disorder [43]. Woman's self-reported mental health history was treated as a binary variable with possible responses 'Yes' or 'No'. Women were classified as part of the 'Yes' group if they had at least one of the following mental health problems at the time of their maternity booking for the index pregnancy: 1) A history of schizophrenia, bipolar affective disorder, depression or any other psychotic illness; 2) A history of postpartum psychotic illness (for multiparous women); 3) A history of inpatient or outpatient treatment by a psychiatrist or mental health team; 4) Self-reported feelings of feeling 'down', depressed or hopeless and/or with 'little interest or pleasure in doing things' during pregnancy (in the past month); 5) A family history of severe mental illness in the postnatal period; 6) A family history of bipolar affective disorder (manic depression). Woman's self-reported mental health history was considered to be 'No' if the woman did not report any of the above conditions.

\section{Potential mediators}

A mediator is a variable "which represents the generative mechanism through which the focal independent variable [i.e., the exposure of interest] is able to influence the dependent variable of interest [i.e., the outcome]" [44], p.1173. Rothman and Greenland [37] suggested that any factor that could be a step in the causal chain between exposure and disease should be treated not as a confounder but as a mediator (an intermediate variable). Several statisticians $[45,46]$ have argued that variables which may act as potential mediators (or variables on the causal pathway to the outcome) should not be adjusted for as this may also adjust away the effect of the exposure of interest ("over-adjustment" [45], p.76).

Based on systematic reviews [10,47], we included a measure of women's perceived control during labour and birth using the Labour Agentry Scale (LAS), in which higher scores indicate greater control [48]. The 
construct validity of the LAS has also been supported by evidence from a series of studies [48-50]. In the current study, the LAS showed good internal consistency reliability with a Cronbach's alpha of 0.82 . We also included neonatal outcomes (gestational age at birth, infant birth weight, infant Apgar score at 1 and 5 minutes, and neonatal intensive care unit admission), medical intervention during labour and birth (mode of birth and manual removal of placenta), and place of birth as potential mediators.

\section{Potential effect modifiers}

Effect modification (which is often termed 'interaction') occurs "when the impact of a risk factor on the outcome is changed by the value of a third variable" [45], p.11. The most central difference between effect modification and confounding is that "whereas confounding is a bias that the investigator hopes to prevent or remove from the effect estimate", effect modification is a real effect and "a property of the effect under study" which the investigator wants to report in the findings [37], p.254.

We included variables to measure social support and perceived stressful events during the 6-8 week postnatal period as potential effect modifiers [45]. Postnatal social support was measured by a woman's living arrangements and the Social Support Scale (SSS), a self-report scale with evidence of sufficient construct validity based on a sample of women from the Avon Longitudinal Study of Pregnancy and Childhood (ALSPAC) [51-54]. To understand women's perceived stress during the postpartum period as a possible consequence of events other than giving birth, we also included the question, "Aside from your birth, have you experienced any changes in your life within the last six weeks, which have caused you anxiety or depression?" If their answer was positive, women were asked to report the event they had experienced. As these variables were measured during the postnatal period, they were not considered as potential confounders. For this reason, they were not included when adjusting for women's baseline characteristics.

\section{Setting and participants}

The site was one of the largest inner city maternity units in England serving a diverse population of women. Women who gave birth under the care of the unit between 7th June and 21st December 2010 were invited to participate. Women who booked to receive their maternity care at the unit could receive obstetric led care (care provided in the main unit, with obstetricians taking primary responsibility for women at high risk of obstetric complications, and midwives taking primary responsibility for women at low risk); care in a midwifery led birth-centre (located in the main unit, with midwives taking primary responsibility for care); or planned home birth (care for by community-based midwives employed by the unit). The inclusion criterion was all women who gave birth after 24 weeks gestation regardless of place of birth, which included women who planned to give birth at the unit but had an unplanned home birth. Exclusion criteria were women unable to read or understand English, women under 16 years old and those who experienced a stillbirth or neonatal death. ${ }^{\mathrm{a}}$ Full ethics and R\&D approval were obtained from the NHS Research Ethics Committee (REC 10/H0772/15) and the study site.

The sample size was based on PTSD symptoms measured by a total score of 20 or more on both the intrusion and avoidance subscales, which was one of the secondary outcomes. We considered all of the four dimensions of PTSD symptoms ( $\geq 20$ on the IES intrusion subscale, $\geq 20$ on the avoidance subscale, $\geq 20$ on either the intrusion or avoidance subscales, and $\geq 20$ on both the intrusion and avoidance subscales) to be important. Thus, it was essential to have a sufficient sample size to give $80 \%$ power for the detection of a significant (at the $5 \%$ level) difference in PTSD symptoms among women who did or did not experience severe morbidity for all of the dimensions of PTSD symptoms. PTSD symptoms that required a total score of 20 or more on both subscales needed the largest sample size. Therefore, we calculated the sample size based on this outcome. Czarnocka and Slade [8] found that approximately $2 \%$ of women from two hospitals in Sheffield, England, had clinically significant levels of both intrusion and avoidance as measured by the IES ( $\geq 20$ on both subscales) at six weeks postpartum. A Dutch study [3] found that $28 \%$ of women had PTSD symptoms (using a self-reported measurement, the PTSD Symptom Scale) within 2 years following births complicated by severe pre-eclampsia, which is likely to account for the higher percentage. The estimate of the incidence of severe maternal morbidity was based on findings of Waterstone et al. [26], who found that $1.2 \%$ of women in their sample from the South East Thames region of England experienced severe maternal morbidity (defined as eclampsia, severe preeclampsia, HELLP syndrome, severe haemorrhage, severe sepsis, and uterine rupture). Based on these findings, a sample size of 1,585 was required, and allowing for a $50 \%$ loss to followup after excluding ineligible women, a total of 3,170 women who met the inclusion criterion were needed to participate.

\section{Data collection}

Midwives provided a study information package to all women who met the study inclusion criterion before they were discharged home from the postnatal ward. 
Women who gave birth at home were provided with the information package by their community midwives. The package included an invitation letter with a study opt-out sheet and a research information leaflet. Women who did not wish to take part were asked to return the opt-out slip before receiving a questionnaire. All women who did not return an opt-out slip were informed that they could withdraw at anytime during the study. Following cognitive testing [55] with a small number of postnatal women $(n=4)$, information on postnatal outcomes, including PTSD symptoms, depression and other variables-such as women's perceived control during labour and birth and perceived social support and stressful events during the postnatal period-was obtained from a follow-up questionnaire posted to women between 6 and 8 weeks after they gave birth. Women were asked to return the questionnaire with a signed consent form in order to participate in the study. A reminder was sent two weeks after the first mailing.

Information on baseline characteristics, pregnancy, birth and neonatal outcomes (including incidence of severe maternal morbidity) of all women who met inclusion criteria was extracted from electronic inpatient maternity records by the IT manager and consultant midwives in the study site (datasets did not include any personally identifiable data except for a study ID). Data from the maternity records were then merged with data from the postnatal questionnaire. Following this, all identifiable data on women who did not return the postnatal questionnaire or did not provide consent for their maternity records to be accessed were removed. This dataset was used to compare baseline characteristics of respondents and nonrespondents. A separate dataset was then created that only included data from women who gave consent for their maternity records to be accessed.

\section{Statistical analysis}

Data analysis was undertaken using SPSS v.19. Descriptive statistics were obtained on postnatal PTSD symptoms and symptoms of depression at 6-8 weeks postpartum. Postnatal outcomes were initially compared between women with and without severe maternal morbidity using Pearson's chi-square tests, Fisher's exact tests and T-tests as appropriate. Multivariable logistic regression models were developed to examine the relationship between severe maternal morbidity and primary outcomes adjusting for women's baseline characteristics. Following recommendations of Baron and Kenny [44], the mediation analysis first tested the relationship between severe maternal morbidity (exposure) and a potential mediator (ie. perceived control during labour and birth measured by the total score of the LAS, neonatal outcomes, mode of birth and place of birth). Next, the bivariate relationship between the potential mediator and PTSD symptoms was examined. If the potential mediator showed statistical significance with both severe maternal morbidity and PTSD symptoms, multivariable logistic regression models were developed to see if the effect size of severe maternal morbidity on PTSD symptoms disappeared (fully mediated) or were reduced (partially mediated) by adding the potential mediator. Logistic regression models were also used to examine a possible effect modification of postnatal social support and other perceived stressful events respectively, on the relationship between severe maternal morbidity and PTSD symptoms, using interaction terms. If the results did not indicate the presence of effect modification (in other words, interaction terms were not significantly associated with PTSD symptoms), they were treated as potential risk factors and simply adjusted for in the multivariable logistic regressions model without using the interaction term. Pairwise deletion was performed for missing data.

\section{Results}

Figure 1 shows the flow of participants through the study. Of the potentially eligible women $(n=3,533), 24$ women opted out. In total, questionnaires were sent to 3,509 women at $6-8$ weeks after giving birth. Fifty-five women could not be contacted by mail. A total of 1,841 questionnaires were returned, although 17 had to be excluded because they were completed by women who had suffered a stillbirth or miscarriage $(n=5)$, most questions were not completed $(n=2)$ or consent to access clinical records was not provided $(n=10)$. The final response rate was $53 \%(n=1824)$, excluding the 55 women from the denominator (therefore, $52 \%$ of all eligible women). Time of questionnaire completion ranged from 6 to 16 weeks, the majority (74.2\%) completing the questionnaire at $6-8$ weeks postnatally; $93 \%$ completed within 10 weeks.

\section{Sample characteristics and severe maternal morbidity}

Respondents were older, more likely to be primiparous, of white ethnicity and living in less-deprived areas compared to non-responders. There were significantly more instrumental and fewer spontaneous vaginal births (SVD) in respondents than non-respondents although rates of caesarean birth (either elective or emergency) were similar. There were no differences between respondents and nonrespondents in severe maternal morbidity exposure where data were available (Additional file 1).

Of the study respondents, 147 (8.1\%) experienced severe maternal morbidity based on our definition of this (Table 1).

\section{Prevalence of postnatal PTSD symptoms and other psychological outcomes}

Descriptive statistics of each postnatal outcome are presented in Table 2. 


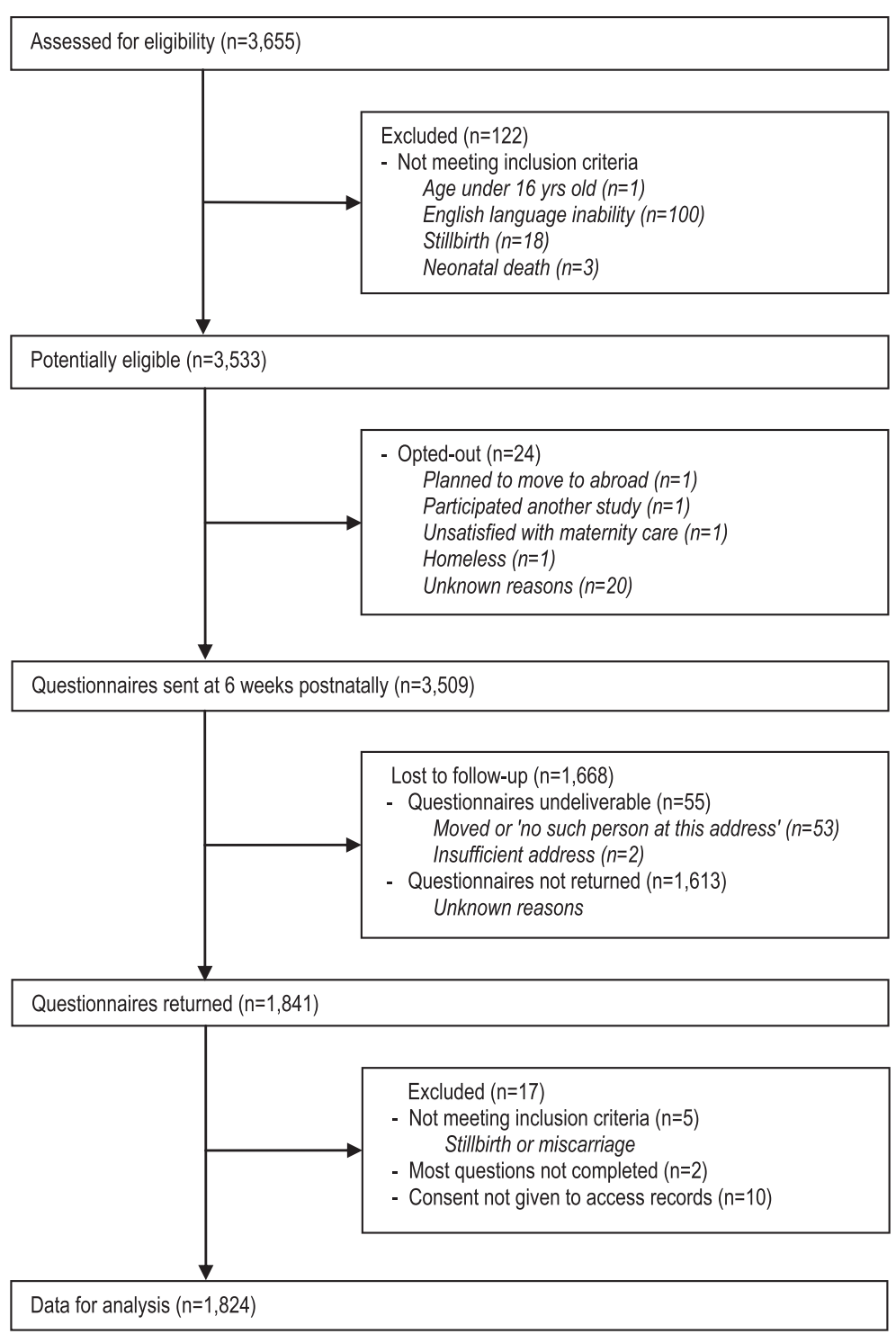

Figure 1 Flow of participants through the study.

PTSD symptoms and other psychological outcomes in women with and without severe maternal morbidity Bivariate analysis showed that the proportion of subjects having PTSD symptoms was statistically significantly higher for women with severe maternal morbidity than women without severe maternal morbidity. The results were consistent for four indicators of PTSD symptoms: (1) intrusion (primary outcome), (2) avoidance (primary outcome), (3) either intrusion or avoidance (secondary outcome), and (4) both intrusion and avoidance (secondary outcome) (see Table 3). The difference in the mean score of the IES was also statistically significant, indicating that women with severe maternal morbidity had more frequent symptoms of intrusion and avoidance at 6 to 8 weeks postpartum. However, no statistically significant differences were observed in either prevalence or severity of depressive symptoms.

\section{Relationship between severe maternal morbidity and PTSD symptoms}

Multivariable logistic regression was developed for the primary outcomes (ie. PTSD symptoms of intrusion and avoidance), following bivariate analysis which examined the relationship of women's baseline characteristics with severe maternal morbidity exposure (Additional file 2) and the outcome (Additional file 3). Results showed that women with severe maternal morbidity had significantly higher odds of having intrusion and avoidance when 
Table 1 Severe maternal morbidity (Respondents $=1,824$ )

Severe maternal morbidity
Major obstetric haemorrhage [25]
Estimated blood loss $\geq 1500 \mathrm{ml}$ (either vaginal or caesarean section related), or transfused 4 or more units of blood during labour,
birth or immediately after birth

Eclampsia [56]

A convulsive condition associated with pre-eclampsia

Severe pre-eclampsia [57]

Pre-eclampsia with an existence of blood pressure of $160 / 110 \mathrm{mmHg}$

HELLP syndrome [25]

Haemolysis (abnormal peripheral blood smear or raised total bilirubin concentration ( $>20.5 \mu$ mol/l)), raised liver enzyme activity (raised aspartate aminotransferase $(>70 \mathrm{U} / \mathrm{I}))$ or raised yglutamyltransferase $(>70 \mathrm{U} / \mathrm{I})$, and low platelets $\left.\left(<100 \times 10^{9} / \mathrm{I}\right)\right)$

Intensive care unit (ICU)/High dependency unit (HDU) admission

ICU/HDU admission after giving birth. Admission for one of the above conditions or for any other reason.

Numbers do not add up to $n=147$ because some women had more than one condition.

compared to women without severe maternal morbidity, even after adjusting for women's baseline characteristics (data not presented).

A series of multivariable logistic regression models were then developed to assess whether the relationship between severe maternal morbidity and PTSD symptoms was mediated by the women's perceived control during labour and birth (measured by the total score of the LAS), infant Apgar score at 5 minutes, mode of birth and place of birth. We selected these four variables following bivariate analysis. The first model (Model 1 in Table 4) shows unadjusted odds ratios for the relationship between severe maternal morbidity and each of the two indicators of PTSD symptoms, intrusion and avoidance. The second model (Model 2 in Table 4) adjusted for clinically important baseline characteristics; age, parity, ethnicity and BMI (potential risk factors of poorer health outcomes), although none met the criteria to be confounders from a statistical point of view. Model 3A in Table 4 showed that the relationship between severe maternal morbidity and PTSD symptoms remained statistically significant $(\mathrm{p}=0.023$ and $<0.001$ for intrusion $\geq 20$ and avoidance $\geq 20$, respectively) once the effect of women's perceived control during labour and birth on PTSD symptoms was removed, although the effect was reduced (from $\mathrm{OR}=2.24$ to 2.04 for $\geq 20$ on IES intrusion subscale; from OR $=3.38$ to 3.15 for $\geq 20$ on avoidance subscale). A similar analysis showed that although better neonatal outcomes (Model 3B) and/or no emergency caesarean birth (Model 3C) slightly reduced the effect of SMM on avoidance symptoms, any mediation effects were partial. There was no evidence that the relationship between SMM and PTSD symptoms was mediated by place of birth (Model 3D). Results consistently showed a direct, statistically significant association between severe maternal morbidity and PTSD symptoms at 6 8 weeks postpartum.

Finally, we developed a multivariable logistic regression model following bivariate analyses to assess the association between severe maternal morbidity and PTSD symptoms, taking into account levels of social support and perceived stressful events during the 6-8 week postnatal period. Since there was no evidence that either of these were effect modifiers (eg. interaction terms were not statistically significant), these factors were simply adjusted for. A statistically significant difference between severe maternal morbidity and PTSD symptoms remained (Table 5). Living arrangements were not included in the model as these were not associated with severe maternal morbidity or PTSD symptoms.

\section{Discussion}

This is one of the largest studies to date to have examined PTSD symptoms among women who have recently given birth and association with severe maternal morbidity. The prevalence of PTSD symptoms at $6-8$ weeks postpartum was within the range estimated from previous studies. Although relatively large numbers of women experienced PTSD symptoms irrespective of severe maternal morbidity, this study found evidence of a higher risk of PTSD symptoms among women who experienced severe maternal morbidity compared with women who did not, in one inner city area of England. The current study also found that a higher level of women's perceived control during labour and birth potentially reduced the effect of severe maternal morbidity on PTSD symptoms. This finding supports the recent synthesis of qualitative studies of women's experiences and perceptions of severe maternal morbidity [47] that showed clinical care and the organisation of care (e.g., communication with 
Table 2 Postnatal outcomes

\begin{tabular}{|c|c|c|c|}
\hline & Frequency & Percentage & $95 \% \mathrm{Cl}$ \\
\hline \multicolumn{4}{|l|}{ Prevalence of PTSD symptoms } \\
\hline \multicolumn{4}{|l|}{ - Intrusion subscale } \\
\hline$<20$ on IES Intrusion & 1669 & $93.6 \%$ & - \\
\hline$\geq 20$ on IES Intrusion & 114 & $6.4 \%$ & $5.3-7.5$ \\
\hline (missing) & (41) & & \\
\hline \multicolumn{4}{|l|}{ - Avoidance subscale } \\
\hline$<20$ on IES avoidance & 1631 & $91.6 \%$ & - \\
\hline$\geq 20$ on IES avoidance & 150 & $8.4 \%$ & 7.1-9.7 \\
\hline (missing) & (43) & & \\
\hline \multicolumn{4}{|l|}{ - Either intrusion $O R$ avoidance subscale } \\
\hline$<20$ on both IES subscales & 1562 & $88.5 \%$ & - \\
\hline$\geq 20$ on either IES Intrusion or $\geq 20$ IES avoidance & 203 & $11.5 \%$ & $10.0-13.0$ \\
\hline (missing) & (59) & & \\
\hline \multicolumn{4}{|l|}{ Both intrusion AND avoidance subscales } \\
\hline$<20$ on at least one IES subscale & 1704 & $96.5 \%$ & - \\
\hline$\geq 20$ on both IES intrusion and $\geq 20$ IES avoidance & 61 & $3.5 \%$ & $2.6-4.3$ \\
\hline (missing) & (59) & & \\
\hline \multicolumn{4}{|l|}{ PTSD symptoms (continuous) } \\
\hline IES intrusion scores & mean $=5.79$ & $\mathrm{sd}=7.33$ & $5.5-6.2$ \\
\hline IES avoidance scores & mean $=5.36$ & $s d=7.79$ & $5.0-5.7$ \\
\hline IES total scores & mean $=11.13$ & $\mathrm{sd}=13.98$ & $10.5-11.8$ \\
\hline \multicolumn{4}{|l|}{ Prevalence of probable depression } \\
\hline EPDS $<13$ & 1,535 & $86.0 \%$ & - \\
\hline$E P D S \geq 13$ & 250 & $14.0 \%$ & $12.4-15.6$ \\
\hline (missing) & (39) & & \\
\hline \multicolumn{4}{|l|}{ Depressive symptoms (continuous) } \\
\hline EPDS total scores & mean $=6.76$ & $s d=5.10$ & $6.53-7.00$ \\
\hline Total & 1,824 & & \\
\hline
\end{tabular}

healthcare professionals) could either mitigate or worsen the negative effects of severe maternal morbidity. These negative effects include women's feelings of loss of control, which can in turn, affect the development of PTSD symptoms. In contrast to PTSD symptoms, there was no evidence of an association between severe maternal morbidity and probable depression as measured using the EPDS, consistent with a previous matched cohort study of severe maternal morbidity conducted with women in the same region [22] and two smaller studies from the Netherlands $[3,58]$. This may be because, unlike PTSD, in which there is almost always a precipitating event [59], depression often occurs without a specific trigger. This is reflected in the DSM-IV, in which PTSD is described as one of only a few mental disorders for which there is a known cause. In contrast, "a diagnosis of depression opens the issue of causation to many factors other than the stated cause of action"
[60], p.297. Therefore any relationship between severe maternal morbidity and depression could be obscured given the many possible causes of depression post-birth.

\section{Strengths and limitations Strengths}

The study design overcame methodological limitations of previous studies such as small sample size $[3,58,61,62]$ and unclear definition of severe maternal morbidity $[2,30,63,64]$. In previous studies with comparatively small samples, the association between SMM and PTSD was often investigated by exploring differences in the mean score of the self-reported measurement of the PTSD symptoms between the risk and non-risk groups. Since the current study had a relatively large sample size, it was possible to compare the proportion of women with a clinically significant level of PTSD symptoms among those 
Table 3 PTSD symptoms and other psychological outcomes in women with and without severe maternal morbidity

\begin{tabular}{|c|c|c|c|c|c|}
\hline & \multicolumn{2}{|c|}{ No severe maternal morbidity } & \multicolumn{2}{|c|}{ Severe maternal morbidity } & \multirow[b]{2}{*}{$\mathbf{P}$} \\
\hline & $\mathrm{N}$, mean & $\%$, sd & $\mathrm{N}$, mean & $\%$, sd & \\
\hline \multicolumn{6}{|l|}{ Prevalence of PTSD symptoms } \\
\hline \multicolumn{6}{|l|}{ - Intrusion subscale } \\
\hline$<20$ on IES Intrusion & 1,541 & $94.1 \%$ & 128 & $88.3 \%$ & $<0.01$ \\
\hline$\geq 20$ on IES Intrusion & 97 & $5.9 \%$ & 17 & $11.7 \%$ & \\
\hline (missing) & (39) & - & (2) & - & \\
\hline \multicolumn{6}{|l|}{ - Avoidance subscale } \\
\hline$<20$ on IES avoidance & 1,518 & $92.7 \%$ & 113 & $79.0 \%$ & $<0.001$ \\
\hline$\geq 20$ on IES avoidance & 120 & $7.3 \%$ & 30 & $21.0 \%$ & \\
\hline (missing) & (39) & - & (4) & - & \\
\hline \multicolumn{6}{|l|}{ - Either intrusion OR avoidance subscale } \\
\hline$<20$ on both IES subscales & 1,454 & $89.6 \%$ & 108 & $75.5 \%$ & $<0.001$ \\
\hline$\geq 20$ on either IES Intrusion or $\geq 20$ IES avoidance & 168 & $10.4 \%$ & 35 & $24.5 \%$ & \\
\hline (missing) & (55) & - & (4) & - & \\
\hline \multicolumn{6}{|l|}{ - Both intrusion AND avoidance subscales } \\
\hline$<20$ on at least one IES subscale & 1,573 & $97.0 \%$ & 131 & $91.6 \%$ & 0.003 \\
\hline$\geq 20$ on both IES intrusion and $\geq 20$ IES avoidance & 49 & $3.0 \%$ & 12 & $8.4 \%$ & \\
\hline (missing) & (55) & - & (4) & - & \\
\hline \multicolumn{6}{|l|}{ PTSD symptoms (continuous) } \\
\hline IES intrusion scores & mean $=5.50$ & $s d=7.16$ & mean $=9.05$ & $s d=8.41$ & $<0.001$ \\
\hline IES avoidance scores & mean $=5.07$ & $s d=7.57$ & mean $=8.71$ & $\mathrm{sd}=9.35$ & $<0.001$ \\
\hline IES total scores & mean $=10.54$ & $\mathrm{sd}=13.61$ & mean $=17.79$ & $\mathrm{sd}=16.33$ & $<0.001$ \\
\hline \multicolumn{6}{|l|}{ Prevalence of probable depression } \\
\hline$<13$ on EPDS & 1,412 & $86.0 \%$ & 123 & $85.4 \%$ & 0.90 \\
\hline$\geq 13$ on EPDS & 229 & $14.0 \%$ & 21 & $14.6 \%$ & \\
\hline (missing) & (36) & - & (3) & - & \\
\hline \multicolumn{6}{|l|}{ Depressive symptoms (continuous) } \\
\hline EPDS total scores & mean $=6.72$ & $\mathrm{sd}=5.11$ & mean $=7.30$ & $\mathrm{sd}=4.90$ & 0.19 \\
\hline Total & 1,677 & & 147 & & \\
\hline
\end{tabular}

with and without severe maternal morbidity. Moreover, in this prospective study, severe maternal morbidity was identified from women's maternity records to minimise recall bias. An additional strength was that the variables potentially on the causal pathway between SMM and PTSD symptoms (ie. women's perceived control during labour and birth, neonatal outcomes, mode of birth, place of birth) were treated as potential mediators. This was important because in previous studies, these variables were simply adjusted for and by doing so the potential effect of severe maternal morbidity might have been eliminated [45].

\section{Limitations}

While avoiding over-adjustment [45] was important, mediation analysis was based on the assumption that severe maternal morbidity might affect PTSD symptoms through potential mediators. However the direction of the relationship between severe maternal morbidity and the mode and place of birth could go either way (eg. mode and/or place of birth might be pre-selected because of severe maternal morbidity or severe maternal morbidity might occur because of mode and/or place of birth). If the latter, then mode and place of birth could be confounders rather than mediators. Because confounders and mediators are statistically very similar, it was not possible to determine the true mechanism of the relationship between severe maternal morbidity and PTSD symptoms. The statistical significance remained the same, however, regardless of whether mode and place-of-birth variables were included in the model. Therefore, the significant association between severe maternal morbidity 
Table 4 Multivariable logistic regression models: association between severe maternal morbidity and PTSD symptoms (mediation analyses)

\begin{tabular}{|c|c|c|c|c|c|c|c|}
\hline & & \multicolumn{3}{|c|}{$\geq 20$ on IES Intrusion subscale } & \multicolumn{3}{|c|}{$\geq 20$ on IES Avoidance subscale } \\
\hline & & ORs & $(95 \% \mathrm{Cl})$ & $\mathbf{P}$ & ORs & $(95 \% \mathrm{Cl})$ & $\mathbf{P}$ \\
\hline \multirow[t]{2}{*}{ Model 1} & SMM (unadjusted) & & & & & & \\
\hline & SMM vs. Non-SMM & 2.24 & $(1.25-4.00)$ & 0.007 & 3.23 & $(2.01-5.17)$ & $<0.001$ \\
\hline \multirow[t]{2}{*}{ Model 2} & SMM (adjusted for potential confounders) ${ }^{\dagger}$ & & & & & & \\
\hline & SMM vs. Non-SMM & 2.23 & $(1.23-4.05)$ & 0.008 & 3.37 & $(2.08-5.46)$ & $<0.001$ \\
\hline \multirow[t]{3}{*}{ Model 3A } & $\mathrm{SMM}^{+}$ & & & & & & \\
\hline & SMM vs. Non-SMM & 2.04 & $(1.10-3.75)$ & 0.023 & 3.14 & $(1.90-5.16)$ & $<0.001$ \\
\hline & Women's perceived control - LAS ${ }^{\dagger}$ (unit = 1 score) & 0.94 & $(0.93-0.96)$ & $<0.001$ & 0.95 & $(0.93-0.96)$ & $<0.001$ \\
\hline \multirow[t]{3}{*}{ Model 3B } & $\mathrm{SMM}^{\dagger}$ & & & & & & \\
\hline & SMM vs. Non-SMM & 2.21 & $(1.22-4.01)$ & 0.009 & 3.28 & $(2.02-5.33)$ & $<0.001$ \\
\hline & 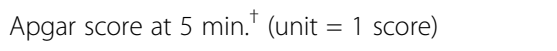 & 0.90 & $(0.72-1.12)$ & 0.34 & 0.76 & $(0.64-0.90)$ & 0.002 \\
\hline \multirow[t]{6}{*}{ Model 3C } & $\mathrm{SMM}^{+}$ & & & & & & \\
\hline & SMM vs. Non-SMM & 2.14 & $(1.15-3.98)$ & 0.017 & 2.55 & $(1.53-4.24)$ & $<0.001$ \\
\hline & Mode of birth ${ }^{\dagger}$ & & & Overall 0.69 & & & Overall $<0.001$ \\
\hline & Assisted vaginal vs. SVD & 0.76 & $(0.40-1.46)$ & 0.42 & 0.94 & $(0.52-1.70)$ & 0.83 \\
\hline & Elective CS vs. SVD & 0.92 & $(0.43-1.94)$ & 0.82 & 1.61 & $(0.88-2.94)$ & 0.12 \\
\hline & Emergency CS vs. SVD & 1.17 & $(0.68-1.99)$ & 0.57 & 2.43 & $(1.56-3.80)$ & $<0.001$ \\
\hline \multirow[t]{6}{*}{ Model 3D } & $\mathrm{SMM}^{\dagger}$ & & & & & & \\
\hline & SMM vs. Non-SMM & 2.16 & $(1.18-3.94)$ & 0.012 & 3.25 & $(1.99-5.29)$ & $<0.001$ \\
\hline & Place of birth ${ }^{\dagger}$ & & & Overall 0.12 & & & Overall 0.31 \\
\hline & AMU vs. OU & 1.05 & $(0.59-1.85)$ & 0.88 & 0.93 & $(0.56-1.53)$ & 0.77 \\
\hline & Planned home vs. OU & - & - & - & 0.32 & $(0.04-2.33)$ & 0.26 \\
\hline & BBA vs. OU & 3.26 & $(1.28-8.33)$ & 0.013 & 1.97 & $(0.78-4.98)$ & 0.15 \\
\hline
\end{tabular}

SMM Severe maternal morbidity, LAS Labour Agentry Scale.

${ }^{\dagger}$ Adjusted for age, parity, ethnic groups, BMI. The results for these variables have been omitted from Models 2, 3A, 3B, 3C and 3D for simplicity of presentations.

- There were too few cases of intrusion to calculate the OR.

Table 5 Multivariable logistic regression model: association between severe maternal morbidity and PTSD symptoms adjusted for potential confounders ${ }^{\dagger}$

\begin{tabular}{|c|c|c|c|c|c|c|}
\hline & \multicolumn{3}{|c|}{$\geq 20$ on IES Intrusion subscale } & \multicolumn{3}{|c|}{$\geq 20$ on IES Avoidance subscale } \\
\hline & ORs & $(95 \% \mathrm{Cl})$ & $\mathbf{P}$ & ORs & $(95 \% \mathrm{Cl})$ & $\mathbf{P}$ \\
\hline \multicolumn{7}{|l|}{ SMM } \\
\hline SMM vs. Non-SMM & 2.21 & $(1.24-3.96)$ & 0.007 & 3.58 & $(2.20-5.84)$ & $<0.001$ \\
\hline \multicolumn{7}{|c|}{ Perceived social support - SSS } \\
\hline (unit = 1 score) & 0.97 & $(0.93-1.00)$ & 0.06 & 0.91 & $(0.88-0.94)$ & $<0.001$ \\
\hline \multicolumn{7}{|c|}{ Perceived stressful event } \\
\hline Yes vs. No & 1.61 & $(0.94-2.77)$ & 0.08 & 1.24 & $(0.75-2.03)$ & 0.40 \\
\hline
\end{tabular}

SMM Severe maternal morbidity, SSS Social Support Scale.

${ }^{\dagger}$ Adjusted for age, parity, ethnic groups, BMI. The results for these variables have been omitted from the model for simplicity of presentation. 
and PTSD symptoms was unlikely to be altered by inclusion of these variables in the model.

Another limitation is that women's perceived control during labour and birth and perceived social support were measured postnatally and it is again difficult to apportion cause and effect. There is a possibility that the association between these variables and PTSD symptoms could be attributed to recall bias in which women with PTSD symptoms were more likely to remember feelings of fear, helplessness, and/or being uncared for during their labour and birth. Similarly, women with PTSD might have felt a lack of support because they needed more support than those with no symptoms. If this was the case, it would be incorrect to include these variables in logistic regression models. The results of multivariable logistic regression analysis with and without these variables, however, did not change the significant association between severe maternal morbidity and PTSD symptoms indicating that study results were unlikely to be affected.

Although higher rates of women's perceived control during labour and birth appeared to reduce the effect of severe maternal morbidity on PTSD symptoms, statistical data itself did not permit an understanding of how much, as it was measured as a continuous score using the LAS. Postnatal outcomes were also collected using a self-administered questionnaire. The measures used were carefully selected, with published accounts of their validity and reliability taken into consideration. Nevertheless, the identification of diagnostic PTSD and depression was not possible in this study. Moreover, PTSD symptoms might vary by the time of questionnaire completion. However due to the small proportion of women who completed the questionnaire very late (> 10 weeks), it was difficult to examine this.

Although we included the women's self-reported mental health histories as collected from maternity booking records, we were unable to measure other maternal characteristics such as previous traumatic events before birth (childhood trauma including previous abuse) and personality type. Individuals with PTSD symptoms might suffer intrusive and distressing memories of past experiences triggered by the current stress event, during which time the individual confused the past stress with present circumstances [65]. Including such information could have been informative.

There is another limitation related to study generalisability. The study included women who had given birth under the care of one large inner city maternity unit and may only be generalisable to the population with similar demographic and obstetric characteristics. The numbers of women who were excluded was small, but it is possible that postnatal health issues were underestimated as a result of excluding potentially high-risk groups. The response rate was $52 \%$ of all eligible women, similar to the response rate in the same region $(51 \%)$ in a recent national maternity survey $[66,67]$. Surveys demand literacy, engagement and organisation, and it was difficult to engage women from younger age groups, poorer areas or different ethnicities in this research, having significant differences between respondents and non-respondents. This is a common issue in research focusing on postnatal population in England as previous studies have shown $[22,66]$.

Due to the lower response from more vulnerable groups and also because women with PTSD symptoms and/or depression might be less likely to respond, there is again a potential risk of the underestimation of psychological problems during the postnatal period as mentioned earlier. However, the results of the significant association between severe maternal morbidity and PTSD were less likely to be affected because the sample was relatively representative in terms of major obstetric haemorrhage (the majority cases of severe maternal morbidity) and none of the indicators for demographic characteristics were likely to be acting as confounders from a statistical point of view.

\section{Further analyses}

It was notable from this study that of the women who did not experience severe maternal morbidity, $5.9 \%$ had a high score on intrusion, $7.3 \%$ on avoidance and $10.4 \%$ had either symptoms. This raises the questions regarding what factors contribute to PTSD symptoms, irrespective of severe maternal morbidity. To answer the question was beyond our original study aims, but will form the basis of a further secondary analysis of this data.

\section{Conclusions}

Despite the concern about increases in the incidence of severe maternal morbidity little was known about the impact on women's psychological health following birth. By conducting a prospective observational study we found clear relationship between women's experiences of severe maternal morbidity and PTSD symptoms at 68 weeks. It is important to raise awareness about the relationship amongst women, clinicians and policy makers in order to prevent and manage severe maternal morbidity and its subsequent issues, and maximising use of finite resources.

In the current UK system of postnatal care, PTSD symptoms among women who have recently given birth may remain 'hidden', for numerous reasons. PTSD is a recent concept, not currently routinely screened for by relevant health professionals. Timely and appropriate treatment of PTSD symptoms may not be offered to women due to the frequent misuse of the term 'postnatal depression' by health professionals as a label for any 
mental illness occurring postnatally [68]. Furthermore women may not report symptoms, due to concerns about social stigma or because they are unaware of the importance of seeking urgent professional support when they experience such symptoms. As recommended in the National Institute for Health and Clinical Excellence [69] guidelines on routine postnatal care, all women should be offered relevant information to recognise symptoms and signs of serious postnatal health problems that they may experience, including PTSD symptoms. Women should also be offered an opportunity to talk about their birth experiences and ask questions about the care they received during labour [69]. These are crucial issues particularly for women who experience severe maternal morbidity who may expect health professionals to help them to make sense of their experiences and the care they received to manage the condition [70].

More studies are required to establish what interventions would increase women's perceived control when emergencies and severe complications occur. However, evidence from qualitative studies show that women feel more in control, even in an emergency situation, when informed about treatment options and involved in decision-making if possible [71,72]. It is therefore important to clearly communicate with women and their partners during an event; respecting their views and providing information and opportunity for them to ask and understand reasons for urgent medical treatment, which may make a difference to the subsequent impact of PTSD symptoms. More research is also needed to consider what care should be included during the shorter and longer-term postnatal period to minimise the impact of severe maternal morbidity as well as analyses of the costeffectiveness of the proposed content and organisation of care.

\section{Ethical approval}

Approval was obtained from the Camden \& Islington Community Research Ethics Committee (REC 10/ H0772/15) and study site. Written consents were also obtained from participants.

\section{Endnote}

${ }^{a}$ The original intention was to include women who suffered a stillbirth or neonatal death because these women were thought to be more vulnerable to psychological problems including PTSD symptoms. During the first two months of recruitment, two women who had experienced a stillbirth unfortunately did not return a study opt-out letter and contacted the researcher expressing concern that they had received a postnatal questionnaire. These two women were excluded. The study team decided to exclude women who had stillbirth or neonatal death given concerns about the distress of being asked to participate. This amendment was approved by the ethics committee on 25 November 2010 (reference number: 10/H0722/15).

\section{Additional files}

Additional file 1: Table S1. Socio-demographic characteristics and pregnancy outcomes (respondents vs. non-respondents).

Additional file 2: Table S2. Bivariate association between women's baseline characteristics and severe maternal morbidity.

Additional file 3: Table S3a. Bivariate association between women's baseline characteristics and PTSD symptoms ( 220 on intrusion subscale of the IES). Table S3b Bivariate association between women's baseline characteristics and PTSD symptoms ( $\geq 20$ on avoidance subscale of the IES).

\section{Competing interests}

The authors declare that they have no competing interests.

\section{Authors' contributions}

MF developed the protocol with the support of DB and JS. All authors contributed to the development of analysis plan. MF collected data with support from DB and JS as well as midwives and IT managers at the study site. MF and DK conducted the main analyses. All authors checked the results. All authors contributed to the manuscript. All authors read and approved the final manuscript.

\section{Acknowledgements}

The authors thank Mrs. Lynne Packanowski, Mrs. Annette Briley and Ms. Mary Sheridan, Dr. Catherine Nelson-Piercy, Mr. Bolaji Coker and Mr. Waqas Shah, the midwives and midwifery managers in the study site for their help in data collection and creating a dataset. Thanks also to Dr. Paul Seed, Dr. Peter Miligan and Dr. Naohiro Yonemoto for their statistical comments at the early stage of the study design. The authors also thank Dr. Kirstie Coxon, Professor Louise Howard, Dr. Lucy Chappell and Dr. Paul Seed for their comments on the draft article.

\section{Author details}

${ }^{1}$ Department of Human Health Sciences, Graduate School of Medicine, Kyoto University, 53 Shogoin Kawara-cho, Sakyo-ku, Kyoto City, Kyoto 606-8507, Japan. ${ }^{2}$ School of Medicine, King's College London, London, UK. ${ }^{3}$ Florence Nightingale School of Nursing and Midwifery, King's College London, London, UK.

Received: 6 September 2013 Accepted: 24 March 2014 Published: 7 April 2014

\section{References}

1. Penney G, Kernaghan D, Brace V: Severe maternal morbidity - the Scottish experience 2003/05. In The Confidential Enquiry into Maternal and Child Health (CEMACH) Saving Mothers' Lives - 2003-2005 The 7th Report on Confidential Enquiries into Maternal Deaths in the United Kingdom. Edited by Lewis G. London: CEMACH; 2007.

2. Creedy DK: Birthing and the development of trauma symptoms: incidence and contributing factors. In Unpublished Ph.D thesis. Griffith University; 1999

3. Engelhard IM, van Rij M, Boullart I, Ekhart THA, Spaanderman MEA, Ma Peeters LLH: Posttraumatic stress disorder after pre-eclampsia: an exploratory study. Gen Hosp Psychiatry 2002, 24(4):260-264.

4. Ayers S, Pickering AD: Do women get posttraumatic stress disorder as a result of childbirth? A prospective study of incidence. Birth-Issues in Perinatal Care 2001, 28(2):111-118.

5. Creedy DK, Shochet IM, Horsfall J: Childbirth and the development of acute trauma symptoms: Incidence and contributing factors. Birth 2000 27(2):104-111.

6. Ryding EL, Wijma B, Wijma K: Posttraumatic stress reactions after emergency cesarean section. Acta Obstet Gynecol Scand 1997, 76(9):856-861.

7. APA: Diagnostic and Statistical Manual of Mental Disorders: DSM-IV. 4th edition. Washington, D.C: American Psychiatric Association; 1994 
8. Czarnocka J, Slade P: Prevalence and predictors of post-traumatic stress symptoms following childbirth. Br J Clin Psychol 2000, 39:35-51.

9. Olde E, van der Hart O, Kleber R, van Son M: Posttraumatic stress following childbirth: A review. Clin Psychol Rev 2006, 26(1):1-16.

10. Furuta M, Sandall J, Bick D: A systematic review of the relationship between severe maternal morbidity and post-traumatic stress disorder. BMC Preg Childbirth 2012, 12(1):125.

11. Ayers S: Thoughts and emotions during traumatic birth: a qualitative study. Birth 2007, 34(3):253-263.

12. Brockington I: Postpartum psychiatric disorders. Lancet 2004, 363(9405):303-310.

13. Halligan SL, Murray L, Martins C, Cooper PJ: Maternal depression and psychiatric outcomes in adolescent offspring: A 13-year longitudinal study. J Affect Disord 2007, 97(1-3):145-154.

14. Kumar RC: "Anybody's child": severe disorders of mother-to-infant bonding. Br J Psychiatry 1997, 171:175-181.

15. Parfitt YM, Ayers $S$ : The effect of post-natal symptoms of post-traumatic stress and depression on the couple's relationship and parent-baby bond. J Reprod Infant Psychol 2009, 27(2):127-142.

16. Hay DF, Pawlby S, Sharp D, Asten P, Mills A, Kumar R: Intellectual problems shown by 1-year-old children whose mothers had postnatal depression. J Child Psychol Psychiatry 2001, 42(7):871-889.

17. Sharp D, Hay DF, Pawlby S, Schmucker G, Allen H, Kumar R: The impact of postnatal depression on boys' intellectual development. J Child Psychol Psychiatry 1995, 36(8):1315-1336.

18. Pawlby S, Sharp D, Hay D, O'Keane V: Postnatal depression and child outcome at 11 years: The importance of accurate diagnosis. $J$ Affect Disord 2008, 107(1-3):241-245.

19. Beck $C T$ : A metaethnography of traumatic childbirth and its aftermath: amplifying causal looping. Qual Health Res 2011, 21(3):301-311.

20. Beck CT, Watson S: Impact of birth trauma on breast-feeding - A tale of two pathways. Nurs Res 2008, 57(4):228-236

21. MacArthur C, Winter HR, Bick DE, Lilford RJ, Lancashire RJ, Knowles $H_{\text {, }}$ Braunholtz DA, Henderson C, Belfield C, Gee H: Redesigning postnatal care: a randomised controlled trial of protocol-based midwifery-led care focused on individual women's physical and psychological health needs. Health Technol Assess 2003, 7(37):1-98.

22. Waterstone M, Wolfe C, Hooper R, Bewley S: Postnatal morbidity after childbirth and severe obstetric morbidity. Int J Obst Gynaecol 2003, 110(2):128-133.

23. Lennox C: Summary of Scottish confidential audit of severe maternal morbidity report 2008. In Saving Mothers' Lives: Reviewing Maternal Deaths to Make Motherhood Safer: 2006-2008: The Eigth Report of the Confidential Enquiries into Maternal Deaths in the United Kingdom. Edited by Lewis G. London: CMACE; 2011:296-198.

24. Lennox C, Marr L: Scottish Confidential Audit of Severe Maternal Morbidity: 7th Annual Report 2009. Healthcare Improvement Scotland: Scotland; 2011.

25. Waterstone $\mathrm{M}$, Bewley $\mathrm{S}$, Wolfe $\mathrm{C}$ : Incidence and predictors of severe obstetric morbidity: case-control study. Br Med J 2001, 322(7294):1089-1093.

26. Zwart JJ, Richters JM, Ory F, de Vries JIP, Bloemenkamp KWM van Roosmalen J: Severe maternal morbidity during pregnancy, delivery and puerperium in the Netherlands: a nationwide population-based study of 371000 pregnancies. Int J Obstetrics Gynaecol 2008, 115(7):842-850.

27. Horowitz M, Wilner N, Alvarez W: Impact of event scale - measure of subject stress. Psychosom Med 1979, 41(3):209-218.

28. Wohlfarth TD, van den Brink W, Winkel FW, ter Smitten M: Screening for Posttraumatic Stress Disorder: an evaluation of two self-report scales among crime victims. Psychol Assess 2003, 15(1):101-109.

29. Coffey SF, Gudmundsdottir B, Beck JG, Palyo SA, Miller L: Screening for PTSD in motor vehicle accident survivors using the PSS-SR and IES. J Trauma Stress 2006, 19(1):119-128.

30. Ayers S: Post-traumatic stress disorder following childbirth. In Unpublished Ph.D thesis. University of London; 1999.

31. Slade P: Towards a conceptual framework for understanding posttraumatic stress symptoms following childbirth and implications for further research. J Psychosom Obstet Gynecol 2006, 27(2):99-105.

32. Horowitz M: Stress response syndromes and their treatment. In Hundbook of Stress: Theoretical and Clinical Aspects. Edited by Goldberger L, Breznitz S. New York: The Free Press; 1982:711-732.
33. Cox JL, Holden JM, Sagovsky R: Detection of postnatal depression. Development of the 10-item Edinburgh Postnatal Depression Scale. $\mathrm{Br}$ Psychiat J Mental Sci 1987, 150:782-786.

34. Murray L, Carothers AD: The validation of the Edinburgh Postnatal Depression Scale on a community sample. Br J Psychiat J Mental Sci 1990, 157:288-290.

35. Zubaran C, Schumacher M, Roxo MR, Foresti K: Screening tools for postpartum depression: validity and cultural dimensions. African $J$ Psychiat 2010, 13(5):357-365.

36. Katz MH: Study Design and Statistical Analysis: A Practical Guide for Clinicians. Cambridge: Cambridge University Press; 2006.

37. Rothman KJ, Greenland S: Modern Epidemiology. 2nd edition. Philadelphia: Lippincott Williams \& Wilkins; 1998.

38. Department for Communities and Local Government: English Indices of Deprivation. 2010, 1-22. http://www.communities.gov.uk/documents/ statistics/pdf/1871208.pdf

39. CMACE: Saving Mothers' Lives: reviewing maternal deaths to make motherhood safer: 2006-08. The Eighth Report on Confidential Enquiries into Maternal Deaths in the United Kingdom. Int J Obstet Gynaecol 2011, 118(Suppl 1):1-203.

40. Kim SY, Dietz PM, England L, Morrow B, Callaghan WM: Trends in prepregnancy obesity in nine states, 1993-2003. Obesity 2007, 15(4):986-993.

41. Knight M: Preeclampsia: increasing incidence but improved outcome? Am J Hypertension 2008, 21(5):491

42. van Roosmalen J, Zwart J: Severe acute maternal morbidity in highincome countries. Best Pract Res Clin Obstet Gynaecol 2009, 23(3):297-304.

43. Simon GE, Von Korff M, Saunders K, Miglioretti DL, Crane PK, van Belle G, Kessler RC: Association between obesity and psychiatric disorders in the US adult population. Arch Gen Psychiat 2006, 63(7):824-830.

44. Baron RM, Kenny DA: The moderator-mediator variable distinction in social psychological research: conceptual, strategic, and statistical considerations. J Personality Soc Psychol 1986, 51(6):1173-1182.

45. Katz MH: Multivariable Analysis: A Practical Guide for Clinicians. 2nd edition. Cambridge: Cambridge University Press; 2006.

46. Rothman KJ, Greenland S, Lash TL: Modern Epidemiology. 3rd edition. Philadelphia, Pa, London: Lippincott Williams \& Wilkins; 2008.

47. Furuta M, Sandall J, Bick D: Women's perceptions and experiences of severe maternal morbidity - A synthesis of qualitative studies using a meta-ethnographic approach. Midwifery 2014, 30(2):158-169.

48. Hodnett ED, Simmons-Tropea DA: The Labour Agentry Scale: psychometric properties of an instrument measuring control during childbirth. Res Nurs Health 1987, 10(5):301-310.

49. Hodnett ED, Osborn RW: A randomized trial of the effects of monitrice support during labor: mothers' views two to four weeks postpartum. Birth-Issues Perinatal Care 1989, 16(4):177-183.

50. Hodnett ED, Osborn RW: Effects of continuous intrapartum professional support on childbirth outcomes. Res Nurs Health 1989, 12(5):289-297.

51. Baker D, Taylor H: The relationship between condition-specific morbidity, social support and material deprivation in pregnancy and early motherhood. Soc Sci Med 1997, 45(9):1325-1336.

52. O'Connor TG, Hawkins N, Dunn J, Thorpe K, Golding J, Team AS: Family type and depression in pregnancy: Factors mediating risk in a community sample. J Marriage Fam 1998, 60(3):757-770.

53. O'Connor TG, Pickering K, Dunn J, Golding J, Team AS: Frequency and predictors of relationship dissolution in a community sample in England. J Fam Psychol 1999, 13(3):436-449.

54. O'Connor TG, Thorpe K, Dunn J, Golding J, Team AS: Parental divorce and adjustment in adulthood: Findings from a community sample. J Child Psychol Psychiat Allied Discipl 1999, 40(5):777-789

55. Willis GB: Cognitive Interviewing: A Tool for Improving Questionnaire Design Thousand Oaks, Calif; London: Sage Publications; 2005.

56. Royal College of Obstetricians and Gynaecologists (RCOG): The Management of Severe Pre-Eclampsia/Eclampsia. London: RCOG press; 2006.

57. NICE: Hypertension in Pregnancy: The Management of Hypertensive Disorders During Pregnancy, Clinical guideline; 107. London: National Institute for Health and Clinical Excellence; 2010

58. Baecke M, Spaanderman ME, van der Werf SP: Cognitive function after pre-eclampsia: an explorative study. J Psychosomatic Obstet Gynecol 2009, 30(1):58-64

59. Rachman S, Brewin C, Dawson B: Anxiety. 2nd edition. Hove: New York: Psychology Press; Taylor \& Francis; 2004 
60. Sparr LF: Forensic issues associated with post-traumatic stress disorder: Twenty-five years later. In Explorations in Criminal Psychopathology: Clinical Syndromes with Forensic Implications. 2nd edition. Edited by Schlesinger LB. Springfield, III: Charles C. Thomas, Publisher; 2007:297-327.

61. Stramrood C, Wessel I, Doornbos B, Aarnoudse J, van den Berg P, Schultz WW, van Pampus M: Posttraumatic stress disorder following pre-eclampsia and PPROM; A prospective study with 15 months follow-up. J Psychosomatic Obstet Gynecol 2010, 31:96-96.

62. Stramrood CAl, Paarlberg KM, In't Veld EMJH, Berger LWAR, Vingerhoets AJJM, Schultz WCMW, Van Pampus MG: Posttraumatic stress following childbirth in homelike- and hospital settings. J Psychosomatic Obstet Gynecol 2011, 32(2):88-97.

63. Lev-Wiesel R, Chen R, Daphna-Tekoah S, Hod M: Past traumatic events: Are they a risk factor for high-risk pregnancy, delivery complications, and postpartum posttraumatic symptoms? I Womens Health 2009, 18(1):119-125.

64. Sorenson DS, Tschetter L: Prevalence of negative birth perception disaffirmation, perinatal trauma symptoms, and depression among postpartum women. Perspect Psychiat Care 2010, 46(1):14-25.

65. Bryant R: Treating the full range of posttramatic reactions. In Clinician's Guide to Posttraumatic Stress Disorder. Edited by Rosen GM, Frueh BC, Hoboken NJ. Chichester: Wiley; John Wiley distributor; 2010:xiv. 295.

66. Care Quality Commission and Picker Institute Europe: Maternity Services Survey. 2010. http://www.cqc.org.uk/sites/default/files/media/documents/ 20101201_mat10_briefing_final_for_publication_201101072550.pdf.

67. Quality Health: Listening to patients: Guy's and St Thomas' NHS Foundation Trust National Maternity Survey management report; 2010.

68. Lewis GM, Drife JO: Why Mothers Die 2000-2002: The 6th Report of the Confidential Enquiries into Maternal Deaths in the United Kingdom. London: Royal College of Obstetricians and Gynaecologists; 2004.

69. National Institute for Health and Clinical Excellence: Routine Postnatal Care of Women and their Babies. London: National Institute for Health and Clinical Excellence; 2006

70. Mapp T: Feelings and fears post obstetric emergencies, part 2. Br J Midwifery 2005, 13(1):36-40.

71. Elmir R, Schmied V, Jackson D, Wilkes L: Between life and death: women's experiences of coming close to death, and surviving a severe postpartum haemorrhage and emergency hysterectomy. Midwifery 2012, 28(2):228-235.

72. Mapp T, Hudson K: Feelings and fears during obstetric emergencies, part 1. Br J Midwifery 2005, 13(1):30-35.

doi:10.1186/1471-2393-14-133

Cite this article as: Furuta et al.: The relationship between severe maternal morbidity and psychological health symptoms at 6-8 weeks postpartum: a prospective cohort study in one English maternity unit. BMC Pregnancy and Childbirth 2014 14:133.

\section{Submit your next manuscript to BioMed Central and take full advantage of:}

- Convenient online submission

- Thorough peer review

- No space constraints or color figure charges

- Immediate publication on acceptance

- Inclusion in PubMed, CAS, Scopus and Google Scholar

- Research which is freely available for redistribution 